\title{
Sequence Labeling with Reinforcement Learning and Ranking Algorithms
}

\author{
Francis Maes, Ludovic Denoyer, and Patrick Gallinari \\ LIP6 - University of Paris 6
}

\begin{abstract}
Many problems in areas such as Natural Language Processing, Information Retrieval, or Bioinformatic involve the generic task of sequence labeling. In many cases, the aim is to assign a label to each element in a sequence. Until now, this problem has mainly been addressed with Markov models and Dynamic Programming.

We propose a new approach where the sequence labeling task is seen as a sequential decision process. This method is shown to be very fast with good generalization accuracy. Instead of searching for a globally optimal label sequence, we learn to construct this optimal sequence directly in a greedy fashion. First, we show that sequence labeling can be modelled using Markov Decision Processes, so that several Reinforcement Learning (RL) algorithms can be used for this task. Second, we introduce a new $\mathrm{RL}$ algorithm which is based on the ranking of local labeling decisions.
\end{abstract}

\section{Introduction}

Sequence labeling is the generic task of assigning labels to the elements of a sequence. This task corresponds to a wide range of real world problems (e.g. character recognition, user modeling, bioinformatic, or information extraction). We consider here supervised sequence labeling where a user provides a training set of labeled sequences and wants to learn a model able to label new unseen sequences. Training examples consist of pairs $(X, Y)$, where $X \in \mathcal{X}$ is an input sequence of elements $\left(x_{1}, \ldots x_{T}\right)$ and $Y \in \mathcal{Y}$ is the corresponding sequence of labels $\left(y_{1}, \ldots y_{T}\right)$. Each $y_{t}$ is the label that corresponds to element $x_{t}$ and $y_{t}$ belongs to the label dictionary denoted $\mathcal{L}$. For example in handwritten recognition, $X$ is a written word (an image for example) and $\mathcal{Y}$ is the corresponding recognized word, with characters in $\mathcal{L}$.

The sequence labeling problem has mainly been addressed with models that are based on first-order Markov dependencies assumptions upon the label sequence. For example, the basic Hidden Markov Models model considers that a label $y_{t}$ only depends on the previous label $y_{t-1}$ and the corresponding input element $x_{t}$. This assumption allows us to use Dynamic Programming algorithms typically the Viterbi algorithm [1 - for computing the best sequence of labels knowing an input sequence. We would like to point out two important limitations of this approach. At first, such models exclude the use of long-term output dependencies and particularly the use of features defined globally on the whole label sequence. NLP offers many examples of such long-term dependencies 
(e.g. is there yet any verb in the sentence?) that cannot be handled by classical Markovian models such as Hidden Markov Models (HMM) or Conditional Random Fields (CRF). A second important issue is that some sequence labeling tasks consider that labels are structured data, e.g a label may represent a set of features, or a relation to another element. For example, for the dependency parsing task, the aim is to identify relations between words. For these problems, the complexity of Viterbi based inference may be prohibitive. These two points motivate a need to develop fast inference methods for sequence labeling.

Contribution: Recently, a promising new approach has been proposed and has been instantiated in two systems named LaSO [2] and Searn [3] (see part 5] for more details). This approach does not try to model joint or posterior probabilities of sequences, but rather attempts at directly modeling the process of inferring the ideal label sequence. Using the same type of idea, we have developed a new method that builds the label sequence sequentially from any input sequence. It is based on Reinforcement Learning (RL) models and allows us to obtain nice performances with a very low complexity. The contributions of this paper are then threefold:

- First, we formalize the sequence labeling task as a Markov Decision Process (MDP) and then we cast the problem of learning as a Reinforcement Learning problem. This idea will make possible the use of different RL methods for sequence labeling and is new up to our knowledge. In the paper, we will use a specific RL method, namely SARSA, to evaluate this idea. This straightforward application of the MDP / RL framework to sequence labeling incrementally builds the desired label sequence in a sequential order starting from the first element of the sequence. We also propose an alternative method where labels can be predicted in any order. The idea is that labels with stronger confidence should be decided first so that an enriched contextual information could help to reduce the ambiguities when deciding for labels with smaller confidence. Since the method can take into account non local contexts, the more decisions there are, the more informative the context is for future decisions.

- Second, we propose a new RL algorithm based on a ranking method. Current RL algorithms use classification or regression frameworks for estimating the next "optimal" action. However all that is needed here is an ordering of these potential actions which will allow us to choose the best one. This is closer to a ranking problem than it is to regression or classification one. The new algorithm relies on the idea of learning to rank possible labeling actions at each step of the inference process. This approach will be shown to have performances comparable to state-of-the-art sequence labeling models while keeping a much lower complexity.

- Third, our MDP view allows us to compare methods like LaSO or Searn with standard RL algorithms and with our ranking based method. We especially discuss the Sampling strategy of these methods, focussing on ideas from the field of RL that could help for the structured prediction community. 
The paper is organized as follows: general background on MDPs and RL algorithms is provided in section 2, section 3 presents our MDP-based models for label sequence building. Section 4 describes our new ranking based approach for learning in these MDP-models. Section 5 describes related work in sequence labeling. The efficiency of the proposed RL approach is demonstrated on three standard datasets in section 6. Last, in section [7, we compare the different methods discussed in the paper.

\section{Background}

Markov Decision Processes 4 provide a mathematical framework for modeling sequential decision-making problems. They are used in a variety of areas, including robotics, automated control, economics and manufacturing. A MDP is defined by a set of states $S$, a set of actions $A$, a transition function $\delta$, a scalar reward function in $\Re$. It describes an environment in which agents can evolve. At any time $t$, an agent is in a given state $s_{t} \in S$. In each state $s$, it can choose between several actions $A_{s} \subseteq A$. Once the agent has chosen one of those actions $a_{t}$, the transition function computes a new state $s_{t+1}$ and the agent receives the immediate reward $r_{t} \in \Re$. An agent relies on a policy $\pi$ which is a function that maps states to action probabilities. Reinforcement Learning (RL) algorithms attempt to find the policy that maximizes the cumulative reward which corresponds to the total amount of reward the agent receives in the long run. Such a policy is called an optimal policy.

Many RL algorithms consider a value function of each state-action pair given a policy. Informally, this Q-function corresponds to how good it is to select a given action in a given state. In this paper, we approximate the action value function using a linear function [5] :

$$
Q_{\theta}(s, a) \stackrel{\text { approx }}{=} \tilde{Q}_{\theta}(\phi(s, a))=<\theta, \phi(s, a)>
$$

where $\langle.,$.$\rangle is the classical dot product, \theta$ is a vector of parameters and $\phi$ is feature function $\phi: S \times A \rightarrow \Re^{n}$ that transforms state-action pairs into vectors. $\tilde{Q}_{\theta}: \Re^{n} \rightarrow \Re$ is called the action value prediction function. Note that this function could also use non-linear models. In order to demonstrate the straightforward application of RL algorithms to the task of sequence labeling, we used the standard approximate-SARSA(0) algorithm [6], for learning $\tilde{Q}$ which defines the sequence labeling policy.

\section{MDPs for Sequence Labeling}

In this section, we develop models of incremental sequence labeling using the MDP formalism. We consider that the sequence labeling task is solved by an agent that, knowing the input sequence $X$, incrementally builds the corresponding output label sequence $Y$. The MDP defines the environment of this agent. 
We first propose, in part 3.1, a model where the agent labels the sequence starting from the first element and then sequentially chooses the label of the next element. In part [3.2, we propose an original approach to sequence labeling, where the agent will label the elements of the input sequence in an order-free manner.

\subsection{Left to Right Labeling}

In order to show how sequence labeling can be modeled using MDPs, we first present the idea of predicting labels from left to right. In Left to Right labeling, initial states correspond to unlabeled element sequences. At each step $t \in[1, T]$ of the process, where $T$ is the length of the input sequence, the agent selects a label $y_{t}$ corresponding to element $x_{t}$. The process finishes at time $T$ when the whole label sequence has been built. In order to express this with an MDP, we define the state space $S$, the set of actions $A$, the transition function $\delta$, and the rewards.

State space. Since labels can depend on the whole input sequence $X$ and on other labels, our states include both the current $X$ and the current partially labeled sequence $\hat{Y}$. Partially labeled sequences are composed of labels in $\mathcal{L} \cup\{\perp\}$ where $\perp$ means that the corresponding element has not been labeled yet. There is one initial state per input sequence $X: s_{\perp X}=(X,(\perp, \ldots, \perp))$.

Action space and transitions. At time $t$, in state $s_{t}$, we want our agent to select a label for $y_{t}$. The possible actions $A_{s_{t}}$ are simply the possible labels for $y_{t}$ given by $\mathcal{L}$. When such an action is performed, the transition function of the MDP returns the new state of the agent. In our case, the transition consists in adding the selected label $y_{t}$ to the already built partial sequence $\hat{Y}$.

Rewards. Sequence labeling is often evaluated using the Hamming loss which counts the number of wrong labels. Since each action corresponds to a single label prediction, we can directly decompose the Hamming Loss over individual actions. Each time the agent fails to predict the correct label it receives a penalty of 1 . Since reward should be maximized, a penalty of 1 corresponds to a reward of -1 . With this reward, maximizing the total amount of reward is equivalent to minimizing the Hamming loss. Sequence labeling can be evaluated with other loss functions, which eventually are not additively decomposable (e.g. F1-scores). In order to enable learning with any loss, the generic solution is to give the whole reward (the opposite of the loss) at the end of the episode. This corresponds to a more traditional RL configuration where the whole sequence of actions leads to a single reward.

Complexity. The Left to Right (LR) sequence labeling MDP is illustrated in figure1(a). Since inference is greedy, its complexity is the number of steps times the complexity of one step. One step requires to evaluate all actions available in $A_{s}$. The complexity of inference in the LR labeling is thus $O(T|\mathcal{L}|)$ where $|\mathcal{L}|$ is the number of possible labels. This complexity is lower than the usual Viterbi complexity $O\left(T|\mathcal{L}|^{2}\right)$. 


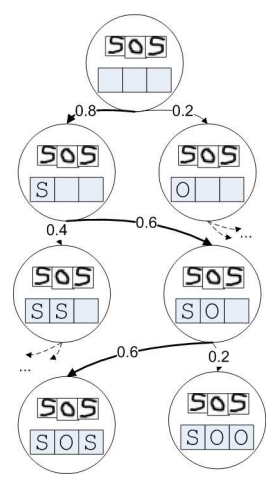

(a) Left to Right

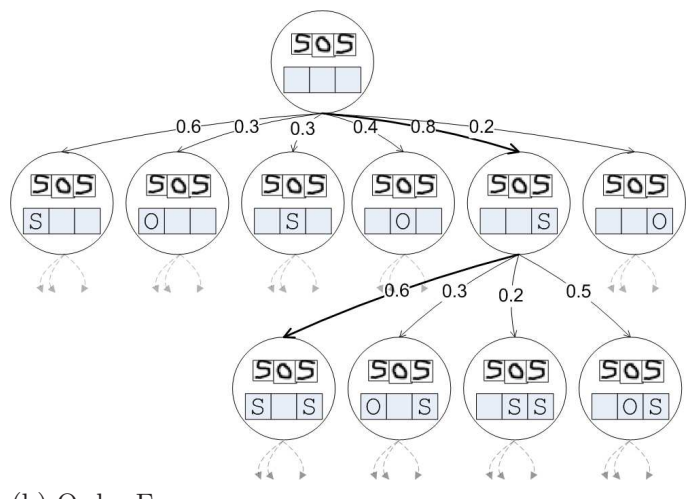

(b) Order Free

Fig. 1. This figure illustrates the Left to Right and Order Free Sequence Labeling MDPs. Each node is a state, which includes both the input sequence $X$ and the partially labeled output sequence $\hat{Y}$. Each edge corresponds to an action and leads to a new state. We have illustrated here, a possible value function $Q(s, a)$ and the corresponding greedy trajectories (bold edges).

\subsection{Order Free Labeling}

Instead of labeling from left to right (or equivalently from right to left), we consider here a model that is able to label in any order. The underyling idea is that it may be easier to first label elements with a high confidence and then to label elements with lower confidence given the labels already chosen. For example, in a handwritten recognition task, some letters may be very noisy whereas others are clear and easy to recognize. If the agent begins to recognize the letters with a high confidence, it then will be able to use these labels, as additional context, to decide how to label the remaining letters. In order to enable order free labeling, an action will consist of both the position $p$ and the label of an element. The action set $A_{s}$ of this new MDP is the cartesian product between $\mathcal{L}$ and the set of unlabeled element positions in $s$. The Order Free (OF) sequence labeling MDP is illustrated in figure 1(b).

Order Free sequence labeling is an original and attracting solution, but it comes with a cost: the number of possible actions per step is much higher than in LR labeling. The inference complexity of OF labeling is $O\left(T^{2}|\mathcal{L}|\right)$. This should be contrasted with the fact that, when the description function $\phi$ only takes into account local dependencies (as with Markov models), a majority of actions remains unchanged when performing one step and their scores do not need to be re-computed at the next step. An efficient implementation could rely on this idea in order to reduce the inference complexity of OF.

\section{Ranking Approach}

In this section, we introduce a new ranking based method for learning the optimal policy in MDPs, such as those presented above. This method will be shown 
to outperform SARSA on the sequence labeling tasks in section 66 Many RL approximate algorithms rely on the idea of modeling the action value function $Q(s, a)$. During inference - the greedy execution of a policy - this value function is used to sort actions and to pick the best one at each step. Since inference only uses an order information in $Q$, we propose here to directly learn to rank actions instead of learning to approximate the value function. This is done by learning an action utility function which defines an order over the set of possible actions.

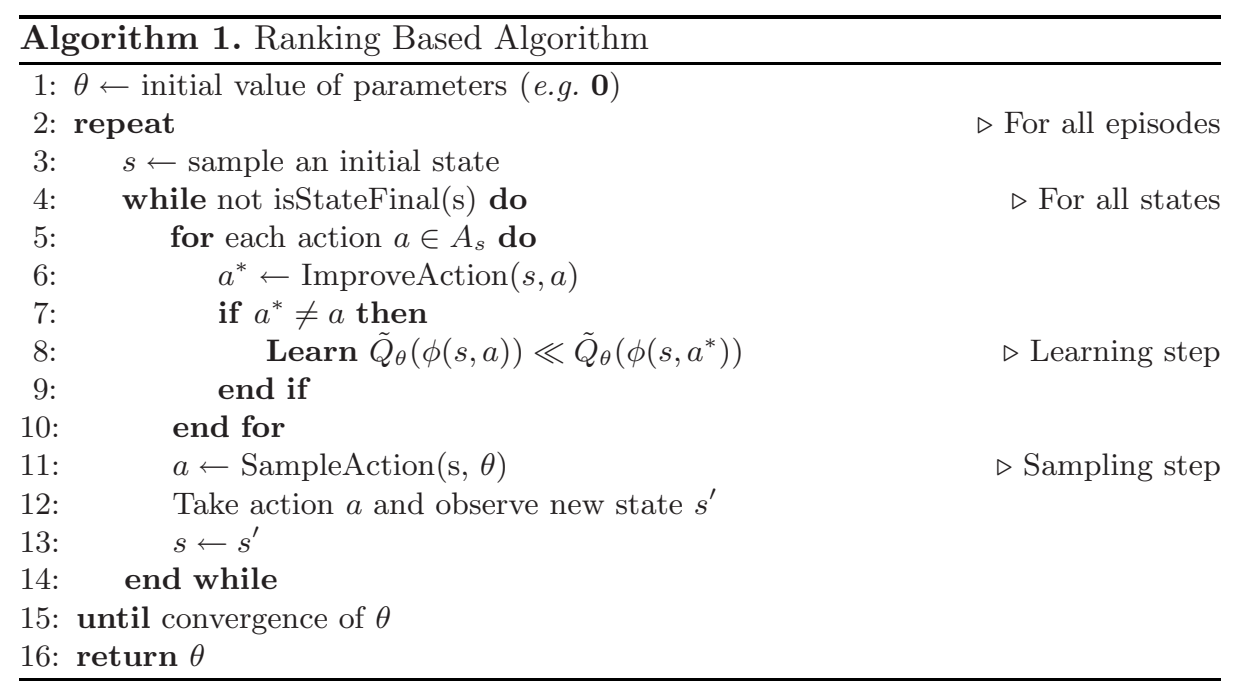

The proposed algorithm (algorithm 1) samples the state-action space in the same way as most RL algorithms do (e.g. with $\epsilon$-greedy sampling, which most of time selects the action with best score, and sometimes selects a random exploratory action). For learning, the algorithm makes the assumption that it can, for any action $a$, get a better action $a^{*}$. This better action is provided through the improvement function ImproveAction $(a)$ (line 6). In models such as LR and OF with Hamming loss, the improvement function is easy to construct: it simply gives the action that provides the best label. In more general situations, the improvement function can be implemented on the basis of simulation using rollout algorithms. We refer the interested reader to [6] and [7] for more information.

For each visited state, the algorithm considers all available actions. For each of those actions $a$, it computes the action $a^{*}=\operatorname{ImproveAction}(a)$ and builds a ranking pair $\left(a, a^{*}\right)$. This pair is then used to update the ranking model (line 9 where the $\ll$ symbol means that utility $\tilde{Q}_{\theta}(\phi(s, a))$ should be made lower than utility $\left.\tilde{Q}_{\theta}\left(\phi\left(s, a^{*}\right)\right)\right)$. Note that any ranking method can be used within algorithm 1, In our experiments, we used a linear utilty function, updated with the online $\tau$-perceptron rule, which is a method close to the standard perceptron update rule that offers better empirical results 8 . 


\section{Related Work}

In this part we present state-of-the-art models for sequence labeling. Some of these models will be used as baselines in the experiments part. Many sequence labeling models based on a Markov assumption over labels have been proposed such as Hidden Markov Models and Conditional Random Fields 9] (CRFs). In such models, a label $y_{t}$ only interacts with the input sequence and its neighborhood labels $y_{t-1}$ and $y_{t+1}$. This enables Dynamic Programming based inference. CRFs model the conditional probability of the label sequence knowing the input sequence, by using the Maximum Entropy principle. Some recent works suggest the use of discriminant training, such as Hidden Markov Support Vector Machine [10] which have been generalized to different structured learning tasks through the SVM ${ }^{\text {struct }}$ model [1]

An other family of methods based on the idea of learning the label sequence building process has started to be explored recently in the structured learning community. The Learning as Search Optimization [2] (LaSO) model learns a scoring function associated to building states. This function is then used to prioritize states using a beam-search procedure. Choosing a beam size of 1 leads to greedy learning and inference methods, which are close to ours. More recently, the same authors have proposed the Searn - Search-Learn - algorithm [3] which reduces the building process to a standard classification task. Searn starts with a good initial policy defined over training examples. The algorithms slowly transforms this initial policy into a fully learned policy able to generalize to new examples. Each learning iteration follows the current policy in order to create a classification example per visited state. These examples are then used to learn a standard classifier. A new policy, which is a linear mixture of the current one and of the new classifier, is then defined for the next iteration. At the end of learning, the initial policy - which only works on training examples - has no more influence on the learned policy. Searn is shown to perform better than LaSO, and also gives better theoretical bounds. See section 7 and 12 for comparison of our approach, LaSO and Searn.

\section{Experiments}

Baseline Models. We have used three baseline methods in order to compare our models: CRFs, SVM ${ }^{\text {struct }}$ and Searn. For CRFs, we used the FlexCRFs implementation 11 with default parameters. We compared with discriminant training of the SVM ${ }^{\text {struct }}$ approach, thanks to the implementation given by the authors: $\mathrm{SVM}^{h m m} 2$. For each dataset, we tried three values of the $C$ parameter: 0.01, 1 , and 100, and kept only the best results. Our last baseline is a simple implementation of Searn provided by the authors. This implementation is limited to sequence labeling with Hamming loss and works with an averaged perceptron as base learner. The implementation does not give access to many parameters, so

\footnotetext{
${ }^{1}$ http://flexcrfs.sourceforge.net

2 http://svmlight.joachims.org/svm_struct.html
} 
few tuning was performed. It should be noted that the authors reports better results than those we present here, when using a Support Vector Machine as base learner.

Data Sets. We performed our experiments on three standard sequence labeling datasets corresponding to three tasks: Spanish Named Entity Recognition (NER3: 9 labels, 10,000 sentences of 50 words), Natural Language Chunking (Chunk: 3 labels, 250,000 tokens ) and Handwritten Recognition (HandWritten [13]: 26 labels, 6,000 words of 5-10 letters). These data sets correspond to the experiments performed in 3] and 11. We used two train/test splits for NER and HandWritten. In the Large split, we used $90 \%$ for training and $10 \%$ for testing and Small is the inverted data set.

Feature Descriptions. All the models we compare rely on a feature function that allows us to jointly describe an $(X, \hat{Y})$ pair. Our approach can use any non-Markovian features but, in order to compare the different methods, we decided to use the same feature information. Due to a lack of space, we do not detail here the features that have been used. The features are described in [12] and are the same than the ones in [3].

\begin{tabular}{|c|cc|cc|ccc|}
\hline & \multicolumn{2}{|c|}{ SARSA } & \multicolumn{2}{c|}{ Ranking } & \multicolumn{3}{c|}{ Baselines } \\
& Left Right Order Free & Left Right & \multicolumn{3}{c|}{ Order Free } & CRF & SVM \\
& struct & Simple Searn $($ LR) \\
\hline NER-small & 91.90 & 91.28 & $\mathbf{9 3 . 6 7}$ & 93.35 & 91.86 & 93.45 & $\mathbf{9 3 . 8}$ \\
NER-large & 96.31 & 96.32 & $\mathbf{9 6 . 9 4}$ & 96.75 & $\mathbf{9 6 . 9 6}$ & - & 96.3 \\
HandWritten-small & 68.41 & 70.63 & 74.01 & 73.57 & 66.86 & $\mathbf{7 6 . 9 4}$ & 64.1 \\
HandWritten-large & 80.33 & 79.59 & 83.80 & $\mathbf{8 4 . 0 8}$ & 75.45 & - & 73.5 \\
Chunk & 96.08 & 96.17 & 96.22 & $\mathbf{9 6 . 5 4}$ & $\mathbf{9 6 . 7 1}$ & - & 95.0 \\
\hline NER-large & $\approx 35 \mathrm{~min}$ & $\approx 11 \mathrm{~h}$ & $\approx 25 \mathrm{~min}$ & $\approx 8 \mathrm{~h}$ & $\approx 8 \mathrm{~h}$ & $>3$ days & $\approx 6 \mathrm{~h}$ \\
HandWritten-large & $\approx 15 \mathrm{~min}$ & $\approx 6 \mathrm{~h}$ & $\approx 12 \mathrm{~min}$ & $\approx 4 \mathrm{~h}$ & $\approx 2 \mathrm{~h}$ & $>3$ days & $\approx 3 \mathrm{~h}$ \\
\hline
\end{tabular}

Results. We compared the LR and OF approaches using both a SARSA learning algorithm and our new ranking algorithm. We also give results of the three baselines described above. We use $\epsilon$-greedy sampling where $\epsilon$ decreases exponentially with the number of iterations. The discount rate in SARSA was tuned manually. Learning rate in both SARSA and our Ranking algorithm decreases linearly. It is not clear whether the OF model helps for better predictions on these datasets. It has been previously shown that first-order dependencies on labels in the NER task do not help much. This could explain why OF and LR are not significantly different on these tasks. In HandWritten and Chunk, OF seams to help a little bit, at the price of a much larger learning time. The ranking approach always perform better than its SARSA counterpart. The idea of ranking actions directly, instead of learning to approximate the value function, leads in our experiments to better generalization. The results demonstrate that our approaches are very competitive for the task of sequence labeling. One can

\footnotetext{
${ }^{3}$ http://www.cnts.ua.ac.be/conll2002/ner

${ }^{4}$ http://www.cnts.ua.ac.be/conll2000/chunking
} 
choose between the fast method: LR, or the method which suffers much less from local ambiguities: OF.

\section{Discussion}

LaSO, Searn, SARSA (and most RL algorithms) or our ranking algorithm are tightly related one to the others. We propose a comparison in 12 which is based on four key points: the sampling strategy, the base learning problem, the feature description strategy and the main learning assumptions each method is based on. We focus here on one major point: the sampling strategy which is the way the actions of the MDPs are chosen during learning. Note that this comparison concerns the use of these methods on general structured prediction tasks and is not specific to sequence labeling.

LaSO: Optimal Sampling. The optimal strategy corresponds to an algorithm that only chooses correct actions. This leads to optimal trajectories: each visited state corresponds to a perfect partial solution. The problem with such trajectories is that the learner is not trained to recover from previous errors. This is very undesirable, since when generalizing, the action chooser will probably do some prediction errors.

Searn: Optimal $\rightarrow$ Predicted Sampling. The Predicted Sampling means that the learner always chooses the action that is predicted by itself at that point. With this kind of sampling, if an error occurs, the learner will be trained to recover from this error. Searn works by moving away from the optimal trajectories toward the predicted trajectories. This has proved to be much more robust than a pure optimal strategy.

Most RL algorithms, Sarsa and Ranking: Predicted + noise Sampling. The Predicted + noise strategy corresponds to a strategy where the agent follows its predicted actions, corrupted with some noise (e.g. with $\epsilon$-greedy or Gibbs sampling). The quantity of noise can be controlled and is generally a decreasing parameter. We believe that this idea from the field of RL should be looked at more closely in the structured prediction community. We believe that this kind of exploration in the state-space leads to more robust learning, especially in the case of few training examples.

\section{Conclusion}

In this paper, we have proposed a new sequence labeling method based on the RL formalism (MDP and SARSA). The key idea proposed here is to model the label sequence building process using a Markov Decision Process. This led us to an original sequence labeling method in which labels can be chosen in any order. We then introduced a Ranking based algorithm in order to efficiently learn how to label a new sequence. Our approach is shown to be competitive with state-ofthe-art sequence labeling methods while being simpler and much faster. Finally, 
on the basis of the link with MDP and RL algorithms, we discussed sampling strategies in LaSO, Searn and RL approaches such as the one we proposed.

We believe that our work is also of general interest for the RL community since it develops an original application of RL algorithms. In order to solve sequence labeling, we construct very large MDPs, where states and actions are formed of structured data. We use a high dimensional feature description of states and actions and present a large scale application of RL which is shown to be competitive with domain specific methods. Furthermore, our application is a successful example of the generalization capability of RL methods.

\section{References}

1. Forney, G.D.: The viterbi algorithm. Proceedings of The IEEE 61(3), 268-278 (1973)

2. Daumé III, H., Marcu, D.: Learning as search optimization: Approximate large margin methods for structured prediction. In: ICML, Bonn, Germany, ACM Press, New York (2005)

3. Daumé III, H., Langford, J., Marcu, D.: Search-based structured prediction (2006)

4. Howard, R.A.: Dynamic Programming and Markov Processes. Technology PressWiley, Cambridge, Massachusetts (1960)

5. J. Si, A. G. Barto, W.B., P., W.II., D.: Handbook of Learning and Approximate Dynamic Programming. Wiley\&Sons, INC., Publications (2004)

6. Sutton, R., Barto, A.: Reinforcement learning: an introduction. MIT Press, Cambridge (1998)

7. Bertsekas, D.P: Rollout agorithms: an overview. In: Decision and Control, pp. 448449 (1999)

8. Tsampouka, P., Shawe-Taylor, J.: Perceptron-like large margin classifiers (2005)

9. Lafferty, J., McCallum, A., Pereira, F.: Conditional random fields: Probabilistic models for segmenting and labeling sequence data. In: ICML, pp. 282-289. Morgan Kaufmann, San Francisco, CA (2001)

10. Altun, Y., Tsochantaridis, I., Hofmann, T.: Hidden markov support vector machines. In: ICML, pp. 3-10. ACM Press, New York (2003)

11. Tsochantaridis, I., Hofmann, T., Joachims, T., Altun, Y.: Support vector machine learning for interdependent and structured output spaces. In: ICML, ACM Press, New York (2004)

12. Maes, F., Denoyer, L., Gallinari, P.: Sequence labeling with reinforcement learning and ranking algorithms. Technical report, LIP6 - University of Paris 6 (2007)

13. Kassel, R.H.: A comparison of approaches to on-line handwritten character recognition. PhD thesis, Cambridge, MA, USA (1995) 